\title{
A CASE OF SUBSTERNAL GOITRE.
}

\author{
By H. V. MORLOCK, M.C., M.D., M.R.C.P.
}

(Physician Miller General Hospital, City of London Hospital for Diseases of the Heart and Lungs, and O. P. Dept., Hampstead General Hospital.)

Miss L. æt. 50 years, seen in consultation with Dr. Holloway, gave the following history. For some years she had suffered from cough and wheeziness, particularly during the winter months. During the last two winters the condition had become more marked and even during the last summer the symptoms had persisted. During the last six weeks the symptoms had become more severe and short attacks of choking and intense dyspncea had appeared. For the last fourteen days these attacks had increased so greatly in severity that during them she became black in the face and partially lost consciousness. In the intervals between the attacks the dyspnoea was severe and distressing and a marked inspiratory and expiratory stridor had appeared. Sleep had become impossible owing to intense dyspnoea and the spasmodic attacks. The pulse had risen steadily up to $\mathrm{I2O}$, while the patient's general condition had steadily deteriorated. There was nothing relevant in the family or past histories.

Condition on Examination. The patient was well covered, lying in the orthopnœic position. Urgent dyspnœe was present and a loud inspiratory and expiratory stridor could be heard. The face, ears, and extremities were markedly cyanosed. Speech was difficult because of the dyspnœa, but phonation was normal. The pupils were equal and slightly dilated, and reacted to light and on accommodation. There was no suggestion of sympathetic nerve stimulation or paralysis. The cranial nerves, throat and tonsils were normal. There were no enlarged glands in the neck, the thyroid was not abnormal to palpation, the veins in the neck were distended, the trachea did not appear to be displaced. Thorax: no distended veins or abnormal pulsation seen. The area of cardiac dullness could not be defined owing to emphysema. Both sides of the thorax were equally distended, the respiratory excursion was poor, the percussion note over both lungs, back and front, was hyperresonant, and no increase in the substernal dullness was detected. On auscultation the air entry was poor but equal over both lungs, back and front. An inspiratory and expiratory stridor could be heard all over both lungs, together with course râles and rhonchi. Vocal resonance was poor but equal in both lungs. There were no glands to be palpated in the axillæ or groins. The abdomen was normal, no enlargement or tenderness of the liver or spleen, no ascites. Legs: no odema: reflexes present and equal. The urine was normal. The larynx was congested but otherwise normal; the cords moved normally. There were no tremors of the outstretched hands.

\section{Commentary.}

The most striking feature of this case was the urgent dyspnœa and marked stridor. This marked stridor made it quite certain that the cause of the urgent dyspnœa was some form of tracheal or bronchial obstruction. Hence such causes of urgent dyspncea as cardiac failure, cardio-renal asthma, and spontaneous pneumothorax could be ruled out because of the stridor and also because of the absence of any confirmatory physical signs of these conditions. It is true that occasionally an enlarged left auricle in mitral stenosis will cause paralysis of the recurrent laryngeal nerve and so give rise to stridor, but again there was no evidence of this. Acute asthma may cause a wheeze but never a true stridor. 
Having decided that the underlying cause was a tracheal or bronchial obstruction, it was necessary to determine its exact nature. The fact that there was no dullness to be found on percussion and that the air entry throughout both lungs was equal, suggested that the obstruction was in the trachea, because, if the obstruction had been in one or other of the main bronchi, the signs or the absence of signs in the thorax would not have been so very definitely symmetrical. The possible causes therefore were:-

I. An inhaled foreign body.

2. Paralysis or œdema of the vocal cords.

3. An aneurysm.

4. A primary intratracheal new growth.

5. A mediastinal new growth.

6. A retrosternal goitre.

As regards I, there was no evidence to suggest a foreign body.

As regards 2, the cords on examination moved freely and were not œdematous and there were no signs of paralysis of any other cranial nerves such as might be expected in an acute or chronic bulbar palsy.

As regards 3, the total absence of all confirmatory signs usually associated with an aneurysm made this diagnosis unlikely.

As regards 4, primary intratracheal new growths are a rarity but the possibility could not be ruled out.

As regards 5, a mediastinal new growth could not definitely be excluded, but the absence of distended veins and percussion change was against it, as also was the length of the history.

As regards 6, a retrosternal goitre was a likely possibility even in the absence of any associated signs of hyperthyroidism. Although an uncommon condition, it is not so rare as a primary intratracheal new growth.

Although the patient appeared moribund it was decided that the most likely diagnosis was a retrosternal goitre or an intratracheal new growth, and as both these conditions were probably amenable to surgical removal, which offered the patient the only hope, it was decided to advise that the risk of transportation be taken and to admit her to the Chest Hospital, Victoria Park, where a bronchoscopic and radiological examination could be undertaken.

On arrival in hospital the chest was $\mathrm{X}$-rayed and the skiagram (Fig. I) showed no evidence of malignant mediastinal tumour or aneurysm, but marked emphysema of both lungs. The small shadow just below the sternal notch was considered to be consistent with the diagnosis of substernal goitre.

Under very light local anæsthesia of the larynx I passed the bronchoscope with the patient in the orthopnoic position, because she could tolerate no other position. On inspection through the bronchoscope a swelling was seen to be pushing into the trachea from the left wall and almost completely occluding the trachea. The swelling was firm but not hard, a catheter was passed down the bronchoscope and past the swelling, and the patient's breathing at once became easier. The bronchoscope was then withdrawn over the catheter and oxygen was administered through the catheter with marked improvement in the patient's condition. 


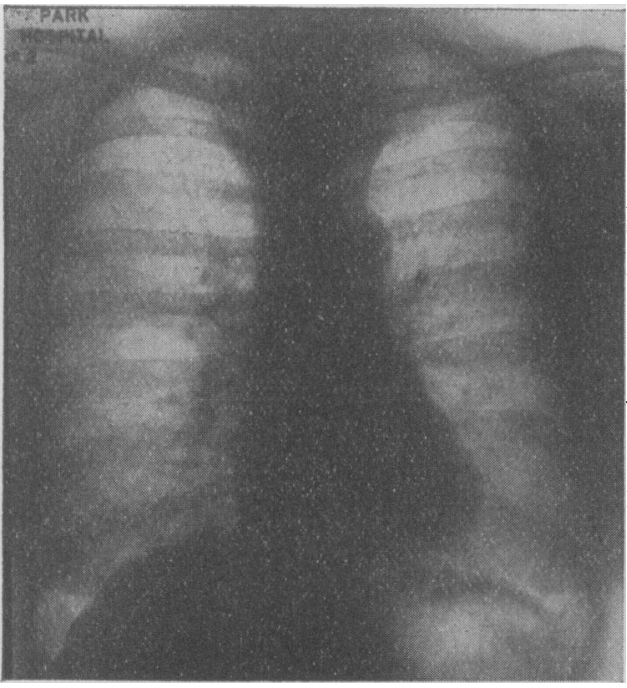

FIG. I. Skiagram taken on day of admission (30.10.35) showing triangular shadow in upper mediastinum.

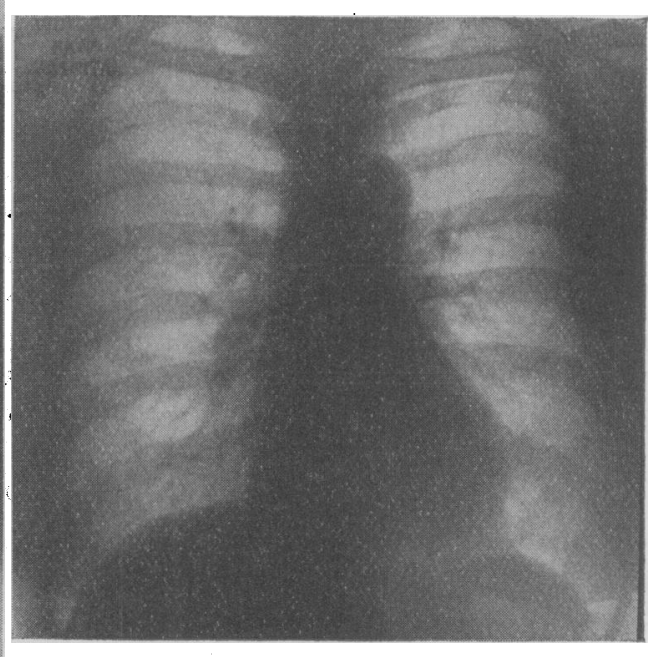

FIG. 2. Skiagram taken on day of discharge (20.11.35) showing absence of shadow in upper mediastinum.

The soft context of the swelling protruding into the larynx suggested a substernal goitre rather than a malignant new growth, and it was decided to advise the patient to take the risk of operation for substernal goitre. If the mass was a sulusternal goitre then one could promise a chance of success, if it turned out to be a new growth then nothing could be promised, but in her condition unless the obstruction was relieved death could only be delayed by a few hours.

The patient decided for operation. Mr. Holmes Sellors was asked to undertake it and agreed to come as soon as possible. A four-hour delay was unavoidable, and during this time the patient was kept fairly comfortable by the administration of oxygen through the catheter. At first the oxygen was administered as fast as possible, but after one hour she developed twitchings and slight carpopedal spasms. The rate of oxygen flow was reduced and the twitchings and spasms ceased. In an acute attack of coughing the patient coughed up the catheter and her condition again became desperate until the catheter was replaced via the bronchoscope with immediate relief.

Mr. Holmes Sellors decided that the patient's condition would not allow of a sternal splitting operation, so under a local anæsthetic, supplemented by morphine and atrophine, and oxygen administered through the catheter, he made a suprasternal incision, and on hooking his finger behind the sternum was able to confirm the diagnosis of a substernal goitre, and in the course of a few moments, during which the patient was in extremis (owing to the manipulation of the trachea) he was able to enucleate the goitre.

On enucleation the relief was immediate and dramatic, and before the patient left the table the catheter was removed and she was able to speak in comfort, and remark on her feeling of well-being. She made an uninterrupted recovery and left hospital in three weeks. Fig. 2 shows the state of matters on X-ray examination just before dismissal, when the small shadow below the sternal notch was no longer visible. 